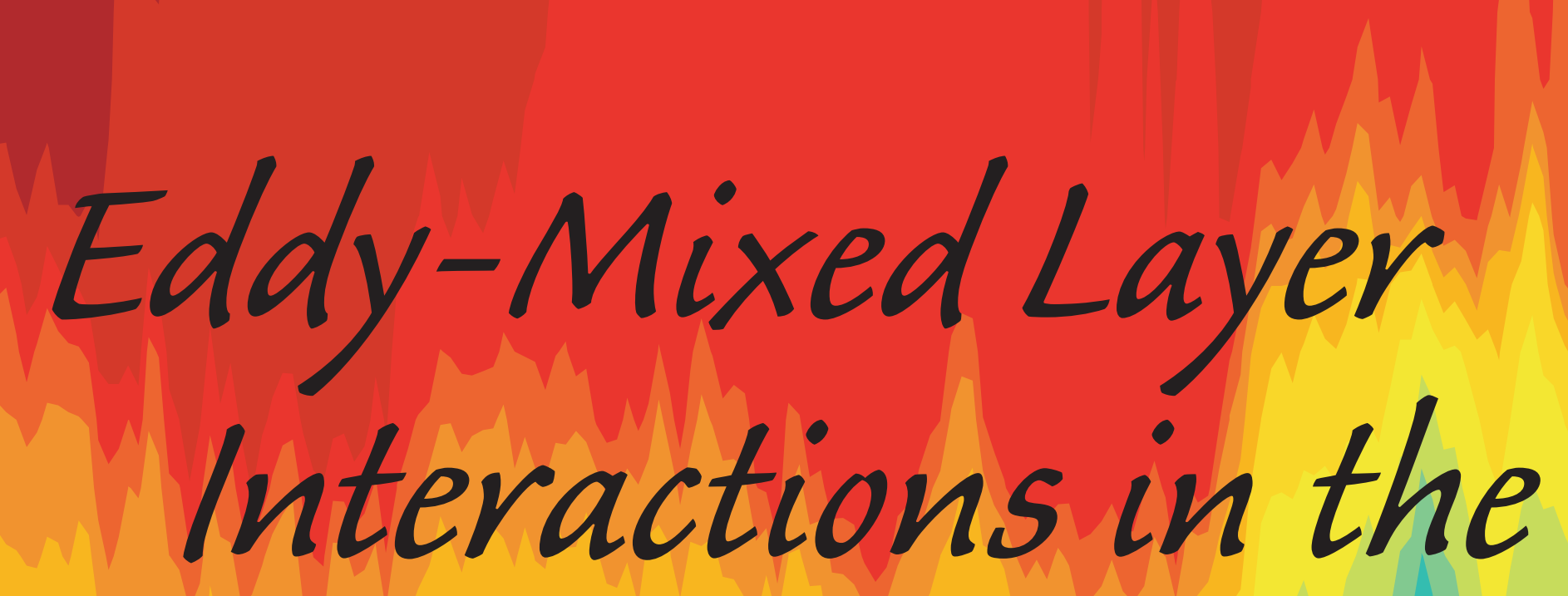

BY RAFFAELE FERRARI AND GIULIO BOCCALETTI 

The mixed layer is a complex dynamical environment that plays host to a large variety of physical phenomena. In broad terms, variability in the surface mixed layer results from a combination of processes arising from three fundamental sources: (1) atmospheric forcing, (2) motions in the ocean interior, (3) and their interactions (Figure 1). The first two are reasonably well understood. The general characteristics of the ocean response to atmospheric forcing-surface fluxes of momentum, heat, salt, and other tracers through the air-sea interfaceare described through one-dimensional models. These models suppose that the properties of the surface layer are set by vertical mixing caused by mechanical stirring from the wind, surface gravity wave breaking, Langmuir circulations, and by convective mechanisms induced by buoyancy loss to the atmosphere (Csanady, 2001). Motions in the ocean interior-the structure and variability of mean currents and mesoscale eddies - are the subject of a vast literature (Gill, 1982). The energy in eddy motions is an order of magnitude larger than that in the mean currents, and dominates the temporal and spatial variability of the ocean circulation. Furthermore, transient motions have been shown to be important in setting the density stratification in the thermocline.

The contributions of atmospheric forcing and mesoscale motions to the upper ocean structure are clearly vis-

Raffaele Ferrari (rferrari@mit.edu) is Victor Starr Assistant Professor of Physical Oceanography, Massachusetts Institute of Technology, Cambridge, MA. Giulio Boccaletti is Postdoctoral Research Associate, Massachusetts Institute of Technology, Cambridge, MA.

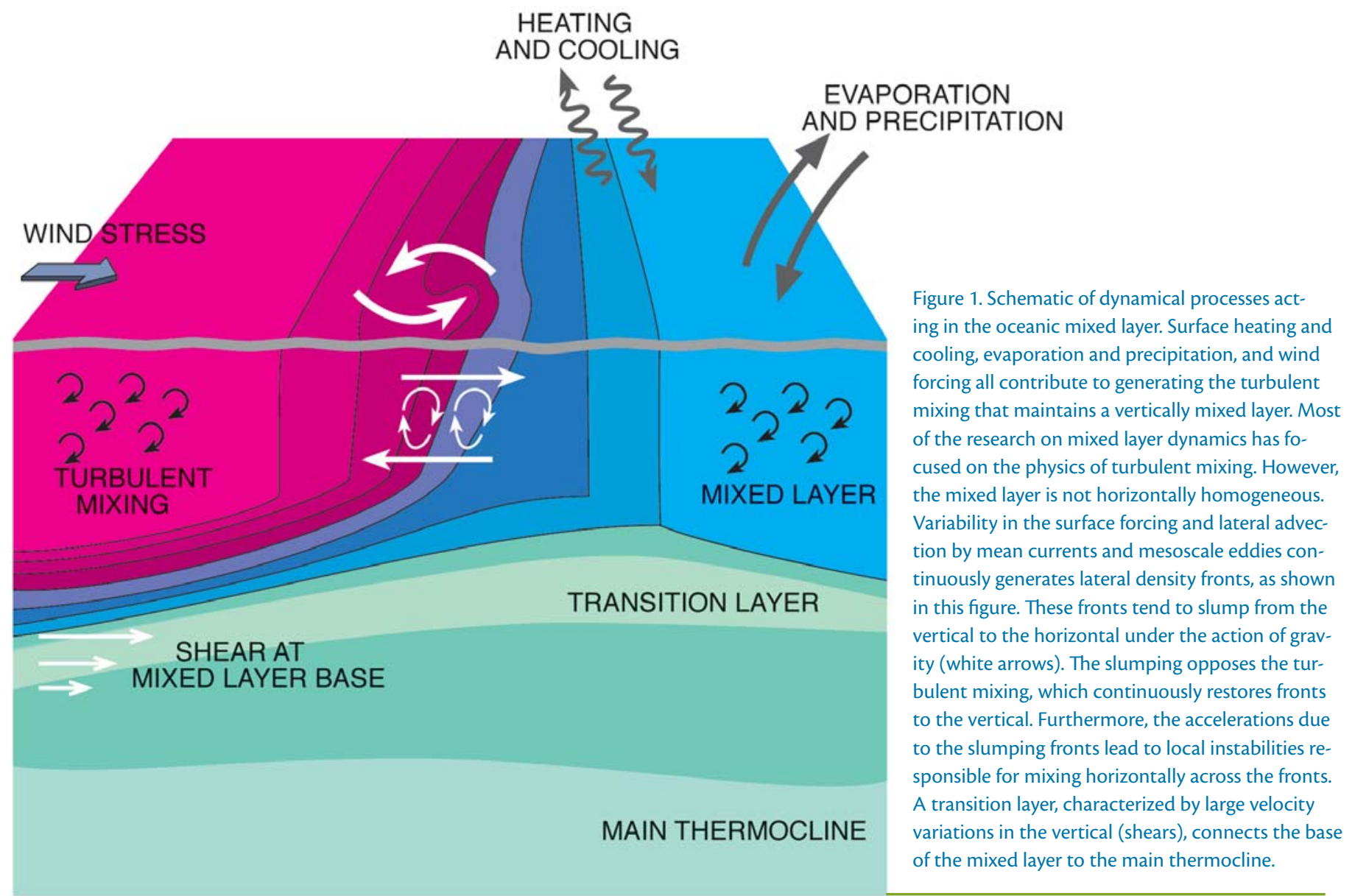




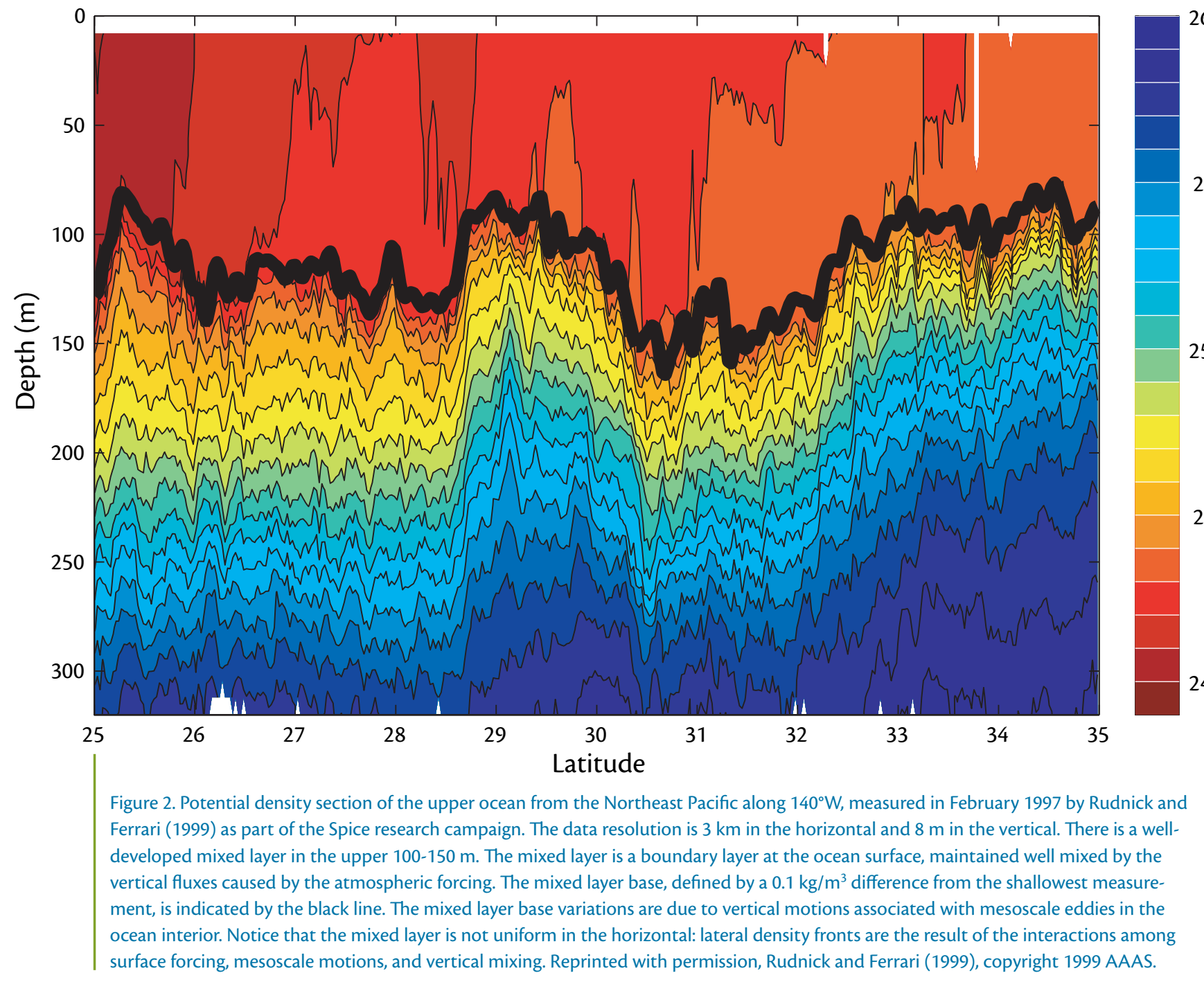

ible in high-resolution surveys. Figure 2 is a section of potential density from the Northeast Pacific $\left(140^{\circ} \mathrm{W}, 25-35^{\circ} \mathrm{N}\right)$ obtained by Rudnick and Ferrari (1999) in February 1997 as part of the oceanographic campaign Spice. Temperature, salinity, and pressure were measured using a conductivity-temperature-depth (CTD) instrument aboard the towed, undulating vehicle SeaSoar. There is a well-developed mixed layer in the up- per $100-150 \mathrm{~m}$, which is vertically mixed by the turbulent fluxes associated with atmospheric forcing. Horizontal fluctuations in mixed layer depth are often mirrored by isopycnal doming below the mixed layer, as occurs in regions between $29^{\circ}$ and $30^{\circ} \mathrm{N}$. This doming is the result of vertical motions associated with deep mesoscale eddies that extend up into the mixed layer.

While the near-surface expression of mesoscale eddies is clear in sections such as that shown in Figure 2, and even in satellite pictures of sea-surface temperature and sea-surface elevation, comparatively little is known about how vertical mixing and the thermocline mesoscale flows interact. This interplay has a twofold impact on upper ocean dynamics: (1) it modulates the air-sea fluxes creating horizontal variability in tracer distributions, in near surface 
stratification, and in mixed layer depth, and (2) it modifies the vertical transport by turbulent motions, therefore affecting exchanges with the oceanic interior (see schematic in Figure 1). These impacts are not negligible and contribute significantly to the budgets of both atmospheric and oceanic properties. Thus, atmospheric forcing and mesoscale motions considered in isolation of each other cannot capture the detailed structure of the mixed layer, nor accurately represent its role in the coupled system. This paper describes some of the recent advances in observing and understanding the physics of that interaction.

\section{MESOSCALE AND SUBMESOSCALE}

\section{DYNAMICS IN THE MIXED LAYER}

Turbulent motions generated by air-sea fluxes maintain the surface boundary layer well mixed in the vertical. Horizontal eddy motions modulate the air-sea interface and introduce lateral variability in the fluxes to and from the atmosphere (Figure 1). Heat fluxes, for instance, depend critically on sea surface temperatures, which are substantially influenced by the advection of anomalously cold or warm water by eddies. The turbulent motions do not act directly on the ensuing temperature anomalies. Rather, the stirring by mesoscale eddies deforms the anomalies into thin filaments. These filaments become unstable, because they are associated with strong horizontal density gradients, and tend to slump. The restratification associated with the slumping interacts in turn with vertical turbulent mixing. Evidence of the slumping instabilities and their interactions with mixing is difficult to produce, because the velocity fields associated with these processes cannot be measured directly in a system as noisy as the oceanic mixed layer. However indirect measurements such as satellite observations, tracer distributions, and data from both moorings and SeaSoar tracks, open a window on the variety of processes present.

The distribution of "tracers" (such as temperature, salinity, nutrient, and oxygen concentrations) offer a useful insight into the properties of horizontal motions in the mixed layer. In particular, the amplitude of tracer fluctuations can be used to infer the structure of the velocity field at different spatial scales. One introduces the tracer spectrum, that is, the tracer variance per unit scale $l$ at different horizontal scales $l$. Thus, the peakto-peak tracer fluctuations at each scale $l$ are given by the spectrum multiplied by $l$. If mesoscale eddies generated in the main thermocline were responsible alone for the stirring in the mixed layer, we would expect the tracer spectrum to decrease with spatial scale as $l^{-1}$ and the corresponding tracer fluctuations to be independent of spatial scale (Salmon, 1998). Instead, observations from instruments towed at a fixed depth in the mixed layer suggest that the spectrum does not decrease with depth on horizontal scales between $100 \mathrm{~km}$ and $10 \mathrm{~m}$, as shown in Figure 3. The corresponding tracer fluctuations grow as $l$ with

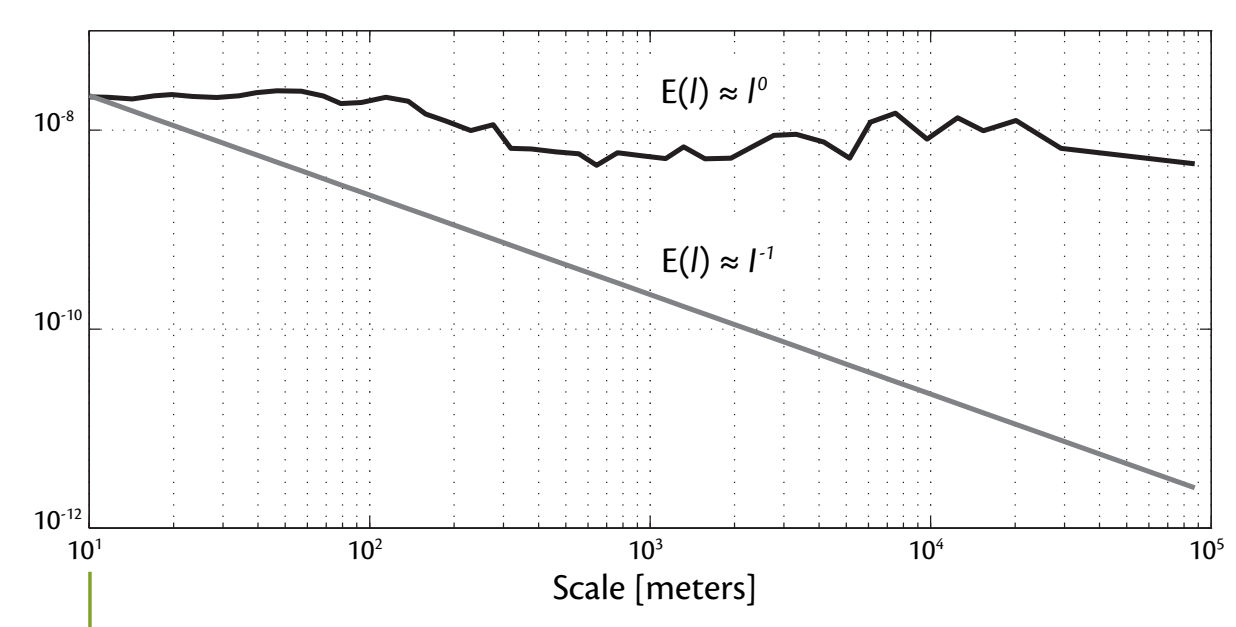

Figure 3. Intensity of temperature fluctuations at different horizontal scales l, as measured by towing a temperature sensor at a fixed depth of $50 \mathrm{~m}$ in the mixed layer shown in Figure 2. The vertical axis represents the variance of temperature fluctuations per unit length scale, $\mathrm{E}(I)$, in ${ }^{\circ} \mathrm{C}^{2} / \mathrm{m}$. The total variance of temperature is given by the integral of the area under $\mathrm{E}(I)$. The variance from data is plotted as a black line and is nearly constant for scales between $10 \mathrm{~m}$ and $100 \mathrm{~km}$. If temperature was stirred only by mesoscale eddies in the surface mixed layer, one would expect $E(I)$ to diminish with scale as shown by the gray line. The discrepancy between the two curves is due to the presence of atmospheric forcing and lateral submesoscale instabilities that interact with the eddy field and modify the temperature distributions. 


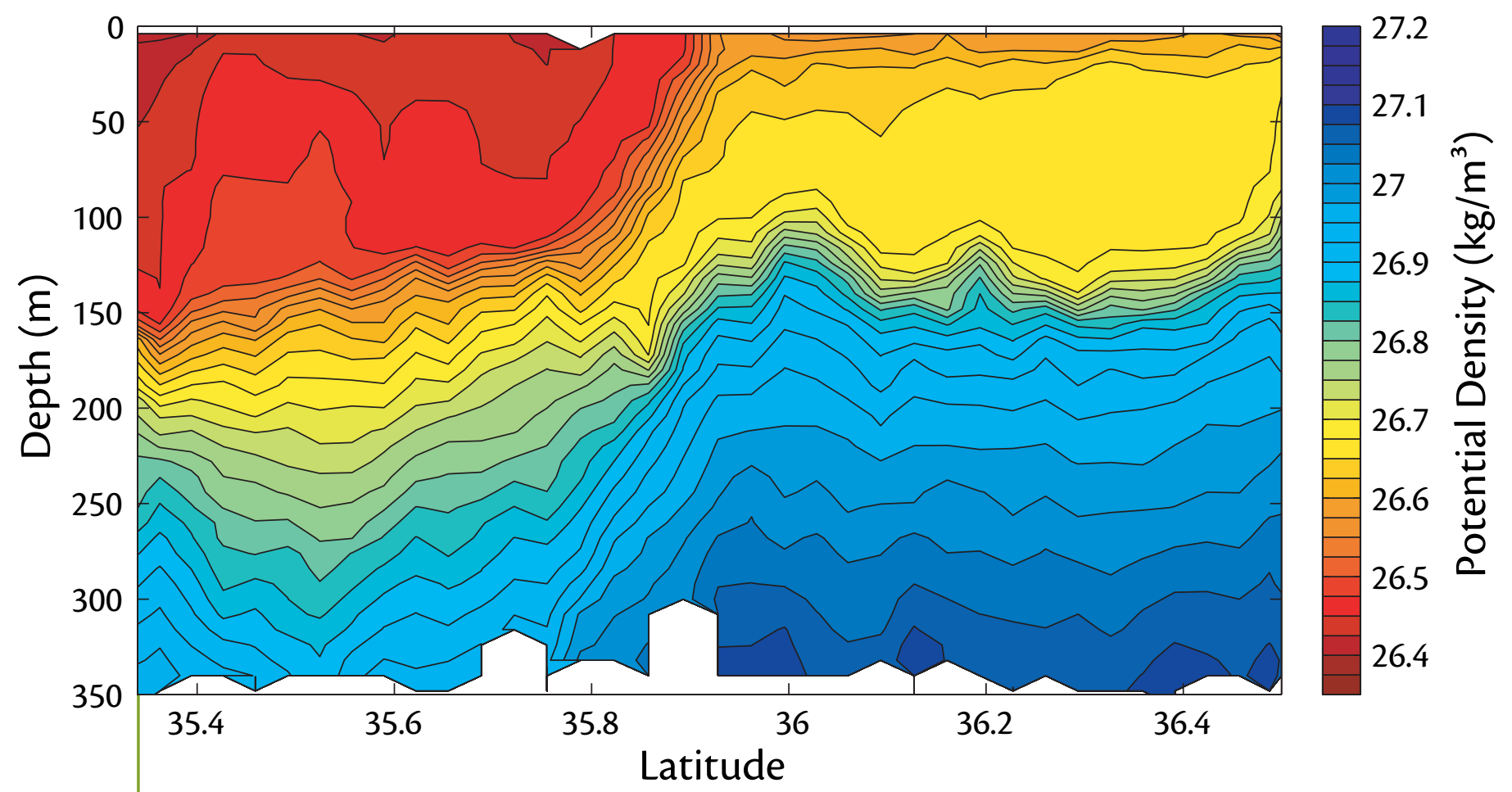

Figure 4. A density section across the Azores Front in the North Atlantic surveyed in March 1992 towing a SeaSoar vehicle equipped with a CTD. Fronts in density, such as the Azores Front, are usually accompanied by hydrodynamical instabilities that tend to slump the front and transport waters across the density gradient. The dynamics of these instabilities play an important role in redistributing tracer properties in the surface mixed layer. Figure from Rudnick and Lutyen (1996).

spatial scale. Therefore mesoscale eddies do not dominate the horizontal dynamics of the mixed layer at all scales, rather submesoscale motions and air-sea fluxes are responsible for stirring and mixing tracers horizontally at scales shorter than $100 \mathrm{~km}$.

A good place to start understanding the nature of submesoscale motions is with frontal structures. Fronts are generated where the mean currents or the mesoscale eddies bring together water masses with different temperatures and salinities. A textbook example is the permanent Azores Front in the North Atlantic shown in Figure 4 (Rudnick and Luyten, 1996). These fronts exhibit hydrodynamic instabilities that manifest themselves in unstable waves and filamentation at the submesoscale. These instabilities are dynamically different from ordinary baroclinic instability—responsible for producing mesoscale eddies-as they occur in an environment with weak density stratification and vigorous vertical mixing. Submesoscale instabilities energize turbulent flows across the lateral density gradients and drive water mass conversion in the mixed layer. This happens through the generation of temperature, salinity, and other tracer gradients at progressively smaller scales, which slump, generating regions of high velocity shear that are ultimately unstable to three-dimensional Kelvin-Helmholtz overturns.

These dynamics are not exclusive to large-scale frontal regions. Instabilities of the kind described above populate scales between $100 \mathrm{~km}$ and a few hundred meters in the mixed layers of the global oceans. Unlike the case of mesoscale eddies, which tend to coalesce and organize themselves into larger scale motions, submesoscale slumping transfers energy to smaller scales so that these motions are critically linked to their ultimate arrest by small-scale, three-dimensional 
turbulent mixing. It is through these processes that the mixed layer mediates the interaction between turbulent mixing and mesoscale eddies.

Evidence that submesoscale motions are ubiquitous comes from a variety of sources. Where surface conditions allow for detection, satellite imagery shows that the ocean is populated with structures at these scales (between $100 \mathrm{~km}$ and $100 \mathrm{~m}$ ) (Munk et al., 2000). Another important and convincing piece of evidence comes from the well-documented phenomenon of density compensation, especially during the winter. The density of seawater is determined by two properties: temperature and salinity. Typically, variations of temperature and salinity generate variations in density. It is, how- ever, possible to have temperature and salinity fronts with no signature in density. This happens if a front is cold and fresh on one side and warm and salty on the other, so that temperature and salinity have opposing effects on density and are said to compensate. One of the most striking features of the winter mixed layer is that temperature and salinity compensate at almost all scales smaller than mesoscale structures. Figure 5 shows temperature and salinity profiles, measured by towing sensors at a fixed depth of $50 \mathrm{~m}$ in the middle of the mixed layer along the whole section shown in Figure 2. Every change in temperature is mirrored by a corresponding change in salinity that compensates its effect on density. This pattern is visible on scales as large as $10 \mathrm{~km}$ and as small as $10 \mathrm{~m}$. The implication is that horizontal processes in the mixed layer work to eliminate horizontal density, but not to eliminate compensated gradients. Temperature and salinity fronts are generated through air-sea fluxes and mesoscale stirring. At some locations these gradients compensate, whereas at other locations they create horizontal density gradients. Horizontal density gradients are unstable and tend to slump, tilting from the vertical to the horizontal. In the winter, turbulence is strong and thus subsequent vertical mixing results in a weakening of the horizontal density gradient. On the other hand, those fronts that are compensated do not slump and are, therefore, stable. The net result of the interaction between

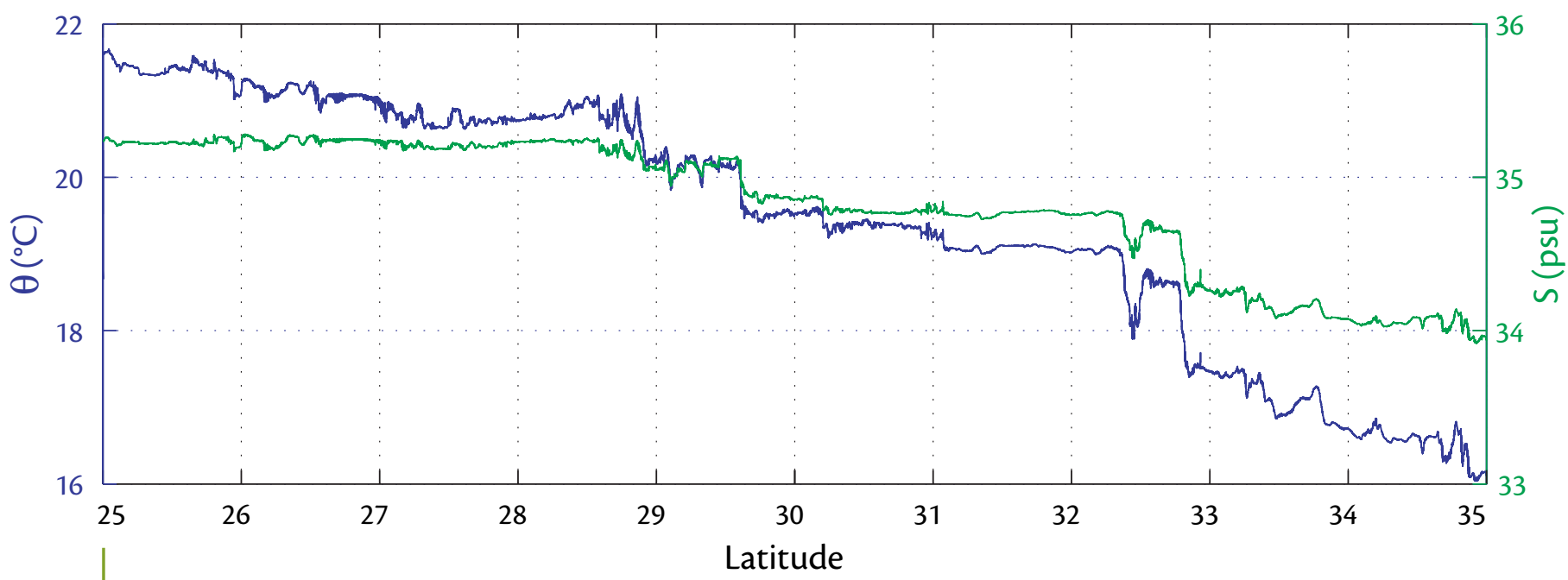

Figure 5. Potential temperature and salinity profiles measured when towing sensors from $25^{\circ} \mathrm{N}$ to $35^{\circ} \mathrm{N}$ at a fixed depth of $50 \mathrm{~m}$ below the surface, right in the middle of the mixed layer shown in Figure 2. The vertical axes are scaled by the thermal and haline expansion coefficients so that equal excursions of temperature and salinity imply identical effects on density. Note that nearly every feature in temperature is mirrored by one in salinity at all scales. Temperature and salinity structure compensate to produce very small density gradients. Reprinted with permission, Rudnick and Ferrari (1999), copyright 1999 AAAS. 
submesoscale instabilities and turbulent vertical mixing is that density fronts diffuse away, while compensated fronts persist much longer.

Although density compensation dominates in winter, during the summer, when atmospheric forcing is weak, the restratification due to slumping fronts can suppress vertical mixing and reduce the mixed layer depth. This leads to further evidence for the importance of submesoscale horizontal motions, as it is known that one-dimensional models tend to overestimate the mixed layer depth in weak mixing conditions, because they neglect the restratification due to lateral instabilities.

Internally generated instabilities only account for part of the horizontal submesoscale motions in the mixed layer. Other notable sources of variabilitywhich we only mention here-include the nonlinear effects associated with the interaction of wind forcing and eddies. The effect of wind forcing, whether it be inertial resonance or lower-frequency Ekman pumping, can be substantially modified by the underlying velocity field produced by the mesoscale eddy field. The modulation of inertial oscillations by the mesoscale eddy field introduces variability in the high-frequency wind-driven shear and in the ensuing shear-driven vertical mixing. The superposition of wind-driven Ekman and mesoscale fields give rise to submesoscale circulations involving significant vertical transports in and out of the mixed layer.

\section{THE TRANSITION LAYER}

The presence of horizontal motions at meso- and submesoscales also modifies the vertical turbulent transfer of properties between the mixed layer and the oceanic interior. The mixed layer base is an important boundary, separating the weakly stratified surface waters from the highly stratified interior (Figure 1). While turbulent mixing does decay with depth, it does not disappear at the mixed layer base, contrary to the traditional notion of the mixed-layer base as a boundary between quiescent and turbulent regions. The transition layer is the region where mixing rates transit from high values in the mixed layer to extremely low values in the interior. This transition region is extremely important for the ventilation of the ocean because all subducted tracers must pass through it, and are modified in it, before joining the geostrophic interior flow. For example, the temperature-salinity structure of the upper ocean differs remarkably from that found in the thermocline waters below. Processes occurring at the mixed layer base are likely to play a significant role in these changes.

Observations show that wind-driven momentum and shear (rate of change of velocity with depth) penetrate below the mixed layer. The penetration depth is typically a few tens of meters, but can occasionally extend to more than a hundred meters. It is an open question as to what determines the thickness of this transition layer: wind-driven shears and instabilities generated in the mixed layer can have a significant projection below and enhance/suppress vertical transports, therefore contributing to the dynamics below. What is clear though is that the transition layer is far from homogeneous in the horizontal. Figure 6 shows the magnitude of the shear in the same upper ocean section used in Figure 2. Shear is very weak in the mixed layer, where turbulent mixing tends to homogenize momentum in the vertical, while it is enhanced below the mixed layer base. There are localized patches where the enhanced shear penetrates down to $300 \mathrm{~m}$, while in other regions the shear penetrates only a short distance below the mixed layer. The penetration depth is not simply proportional to the strength of the local wind field at the surface: horizontal mesoscale and submesoscale motions modify the wind-driven motions as they radiate from the surface.

While shear and mixing processes are often described as one dimensional involving the vertical energy balance of the water column, horizontal eddy motions on a wide range of scales modulate such processes significantly. A passing disturbance, for instance, can temporarily modify the stratification of the water column or the vertical transport, therefore changing the background state upon which the vertical processes act. This might change significantly the rates of water exchanges between the mixed layer and the interior, and affect the global budgets of the exchanged properties. 


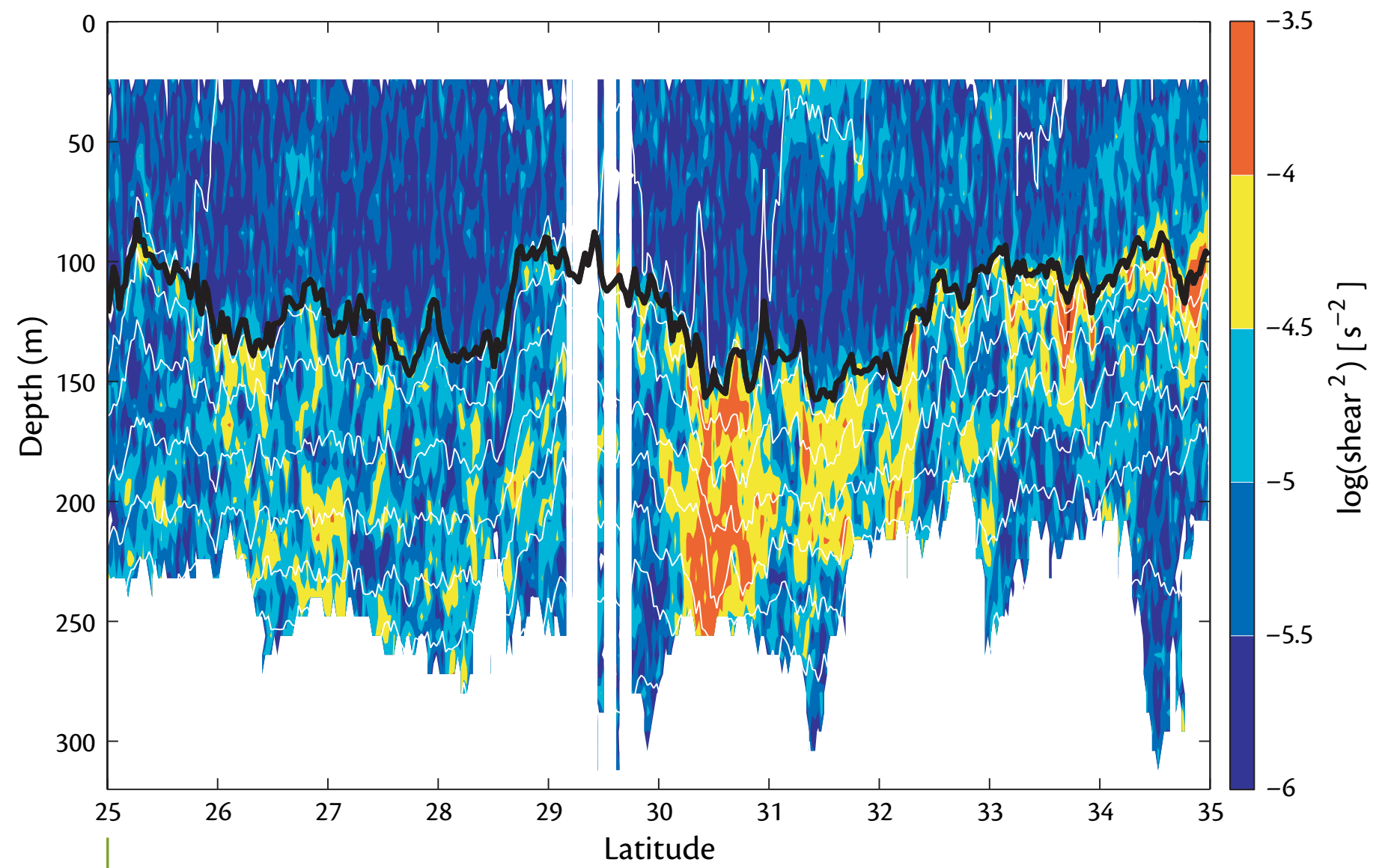

Figure 6. Velocity shear squared for the ocean section shown in Figure 2. The shear is the velocity change per unit vertical distance. The shear shown in this figure is computed across vertical intervals of $8 \mathrm{~m}$. The black line denotes the mixed layer base, defined as a $0.1 \mathrm{~kg} /$ $\mathrm{m}^{3}$ change from the surface value. The shear is very weak in the mixed layer because vertical mixing tends to homogenize momentum and remove any velocity change. Shear is instead high below the mixed layer, in the transition layer. (Notice however that there is some shear in the mixed layer. A comparison of shear and density stratification in the mixed layer shows that density is better homogenized than momentum. This difference is due to Earth's rotation, which generates shears in the presence of horizontal density gradients.) The maximum values of shear are right at the mixed layer base, and only occasionally penetrate deeper than a few tens of meters below this base. The high shears in the transition layer generate density overturns and produce mixing. The mixing rates are, however, smaller than in the mixed layer, and do not eliminate completely the density stratification.

\section{NUMERICAL MODELS OF THE UPPER OCEAN}

This cursory survey of upper ocean dynamics has shown that mesoscale, submesoscale, and turbulent processes interact and control the lateral and vertical transport of tracers and momentum at the ocean surface. Thus, the large-scale circulation in the upper ocean depends crucially on motions with scales between $100 \mathrm{~km}$ and a few meters. Given the limitations of today's computers, numerical models used for climate studies cannot afford to resolve the full range of oceanic motions, and thus must resort to parameterizing the effect of small-scale motions on the larger scales. The challenge is then to find mathematical operators that accomplish the necessary physical effects associated with unresolved smallscale motions, so as to obtain closed 
equations for the large-scale circulation.

In present climate models, the ocean horizontal grid resolution is $\mathrm{O}(100) \mathrm{km}$ or larger. At this resolution, mesoscale and submesoscale eddy dynamics, and turbulent boundary mixing must all be parameterized. A number of parameterization schemes have been proposed to represent the effects of mesoscale eddies in the ocean interior. One-dimensional models have been derived to parameterize air-sea fluxes and boundary-layer turbulence. Unfortunately our poor understanding of the interactions between these two classes of motion has hampered attempts to derive closure schemes that account for both. The common practice is to use parameterizations for mesoscale motions only in the ocean interior, where turbulence is weak and does not affect larger-scale motions. Mesoscale and submesoscale eddies are instead ignored in the boundary layers, where parameterizations account only for turbulent mixing processes. As discussed above, this is at odds with the observational and theoretical evidence that eddy fluxes have a strong impact on upper-ocean dynamics.

Until these issues have been adequately addressed, coupled climate models will be severely limited in simulating air-sea interactions. Some progress is expected in the near future thanks to a project recently sponsored by the National Science Foundation and the National Oceanic and Atmospheric Administration. The project comprises a group of fifteen leading oceanographers organized in a Climate Process Team that works in close collaboration with modeling centers in Princeton and Boulder. The group is using a combination of theory and observations of the kind described above to derive parameterizations of eddymixed layer interactions suited for ocean models used in climate studies. Morepowerful computers will appear in the future and may permit decreasing the horizontal resolution to $\mathrm{O}(10) \mathrm{km}$ in the next decade. At this resolution mesoscale eddies will be partly resolved. However, the interactions between mesoscale eddies and turbulent fluxes in the mixed layer happen at much smaller scales and need to be parameterized even at $\mathrm{O}(10)$ $\mathrm{km}$ resolution. Thus, the problem of understanding and parameterizing eddymixed layer interactions will remain a critical issue for climate studies in the near future.

\section{CONCLUSION}

The oceanic mixed layer plays a fundamental role in climate because it controls the exchange of heat, freshwater, carbon dioxide, and many other properties between the ocean and the atmosphere. It is therefore no surprise that this layer has been the focus of oceanography since the pioneering work of V. Walfrid Ekman at the beginning of last century. (Ekman was a Swedish physical oceanographer best known for his studies of the dynamics of ocean currents and who is credited with recognizing the role of the Coriolis effect on ocean currents.) However, only in the last decade have observations of the three-dimensional structure of the mixed layer become available. Instru- ments mounted on SeaSoar towing vehicles, ocean gliders, floats, drifters, and moorings are shedding light on a wide range of phenomena never observed before, revealing an unexpected complexity. While these data pose a challenge for modelers and theoreticians alike, they are reinvigorating the field as new puzzling pieces of evidence appear with increasing frequency in scientific journals. It is indeed an exciting time for the field, and we hope that many of the issues raised in this article will soon find an explanation through the combined effort of observationalists, numerical modelers, and theoreticians.

\section{ACKNOWLEDGEMENT}

The authors acknowledge the support of the National Science Foundation under awards OCE02-4152 and OCE03-36755.

\section{REFERENCES}

Csanady, G.T. 2001. Air-Sea Interaction. Cambridge University Press, 284 pp.

Gill, A. 1982. Atmosphere-Ocean Dynamics. Academic Press, San Diego, 662 pp.

Munk, W., L. Armi, K. Fischer, and F. Zachariasen. 2000. Spirals in the sea. Proceedings of the Royal Society of London 456(Series A):1217-1280.

Rudnick, D.L., and R. Ferrari. 1999. Compensation of horizontal temperature and salinity gradients in the ocean mixed layer. Science 283:526-529.

Rudnick, D.L., and J.R. Luyten. 1996. Intensive surveys of the Azores Front: 1, Tracers and dynamics. Journal of Geophysical Research 101:923-939.

Salmon, R. 1998. Geophysical Fluid Dynamics. Oxford University Press, New York, 378 pp. 